\title{
Why Johnny Can't Pentest: An Analysis of Black-box Web Vulnerability Scanners
}

\author{
Adam Doupé, Marco Cova, and Giovanni Vigna \\ University of California, Santa Barbara \\ \{adoupe, marco, vigna\}@es.ucsb.edu
}

\begin{abstract}
Black-box web vulnerability scanners are a class of tools that can be used to identify security issues in web applications. These tools are often marketed as "point-and-click pentesting" tools that automatically evaluate the security of web applications with little or no human support. These tools access a web application in the same way users do, and, therefore, have the advantage of being independent of the particular technology used to implement the web application. However, these tools need to be able to access and test the application's various components, which are often hidden behind forms, JavaScript-generated links, and Flash applications.

This paper presents an evaluation of eleven black-box web vulnerability scanners, both commercial and open-source. The evaluation composes different types of vulnerabilities with different challenges to the crawling capabilities of the tools. These tests are integrated in a realistic web application. The results of the evaluation show that crawling is a task that is as critical and challenging to the overall ability to detect vulnerabilities as the vulnerability detection techniques themselves, and that many classes of vulnerabilities are completely overlooked by these tools, and thus research is required to improve the automated detection of these flaws.
\end{abstract}

\section{Introduction}

Web application vulnerabilities, such as cross-site scripting and SQL injection, are one of the most pressing security problems on the Internet today. In fact, web application vulnerabilities are widespread, accounting for the majority of the vulnerabilities reported in the Common Vulnerabilities and Exposures database [4]; they are frequent targets of automated attacks [20]; and, if exploited successfully, they enable serious attacks, such as data breaches [9] and drive-by-download attacks [17]. In this scenario, security testing of web applications is clearly essential.

A common approach to the security testing of web applications consists of using black-box web vulnerability scanners. These are tools that crawl a web application to enumerate all the reachable pages and the associated input vectors (e.g., HTML form fields and HTTP GET parameters), generate specially-crafted input values that are submitted to the application, and observe the application's behavior (e.g., its HTTP responses) to determine if a vulnerability has been triggered.

Web application scanners have gained popularity, due to their independence from the specific web application's technology, ease of use, and high level of automation. (In fact, web application scanners are often marketed as "point-and-click" pentesting 
tools.) In the past few years, they have also become a requirement in several standards, most notably, in the Payment Card Industry Data Security Standard [15].

Nevertheless, web application scanners have limitations. Primarily, as most testing tools, they provide no guarantee of soundness. Indeed, in the last few years, several reports have shown that state-of-the-art web application scanners fail to detect a significant number of vulnerabilities in test applications [1,16,21,22,24]. These reports are valuable, as they warn against the naive use of web application scanners (and the false sense of security that derives from it), enable more informed buying decisions, and prompt to rethink security compliance standards.

However, knowing that web application scanners miss vulnerabilities (or that, conversely, they may raise false alerts) is only part of the question. Understanding why these tools have poor detection performance is critical to gain insights into how current tools work and to identify open problems that require further research. More concretely, we seek to determine the root causes of the errors that web application scanners make, by considering all the phases of their testing cycle, from crawling, to input selection, to response analysis. For example, some of the questions that we want to answer are: Do web application scanners correctly handle JavaScript code? Can they detect vulnerabilities that are "deep" in the application (e.g., that are reachable only after correctly submitting complex forms)? Can they precisely keep track of the state of the application?

To do this, we built a realistic web application, called WackoPicko, and used it to evaluate eleven web application scanners on their ability to crawl complex web applications and to identify the associated vulnerabilities. More precisely, the WackoPicko application uses features that are commonly found in modern web applications and that make their crawling difficult, such as complex HTML forms, extensive JavaScript and Flash code, and dynamically-created pages. Furthermore, we introduced in the application's source code a number of vulnerabilities that are representative of the bugs commonly found in real-world applications. The eleven web application scanners that we tested include both commercial and open-source tools. We evaluated each of them under three different configuration settings, corresponding to increasing levels of manual intervention. We then analyzed the results produced by the tools in order to understand how the tools work, how effective they are, and what makes them fail. The ultimate goal of this effort is to identify which tasks are the most challenging for black-box vulnerability scanners and may require novel approaches to be tackled successfully.

The main contributions of this paper are the following:

- We performed the most extensive and thorough evaluation of black-box web application vulnerability scanners so far.

- We identify a number of challenges that scanners need to overcome to successfully test modern web applications both in terms of crawling and attack analysis capabilities.

- We describe the design of a testing web site for web application scanners that composes crawling challenges with vulnerability instances. This site has been made available to the public and can be used by other researchers in the field.

- We analyze in detail why the web application vulnerability scanners succeed or fail and we identify areas that need further research. 


\section{Background}

Before discussing the design of our tests, it is useful to briefly discuss the vulnerabilities that web application scanners try to identify and to present an abstract model of a typical scanner.

\subsection{Web Application Vulnerabilities}

Web applications contain a mix of traditional flaws (e.g., ineffective authentication and authorization mechanisms) and web-specific vulnerabilities (e.g., using user-provided inputs in SQL queries without proper sanitization). Here, we will briefly describe some of the most common vulnerabilities in web applications (for further details, the interested reader can refer to the OWASP Top 10 List, which tracks the most critical vulnerabilities in web applications [13]):

- Cross-Site Scripting (XSS): XSS vulnerabilities allow an attacker to execute malicious JavaScript code as if the application sent that code to the user. This is the first most serious vulnerability of the OWASP Top 10 List, and WackoPicko includes five different XSS vulnerabilities, both reflected and stored.

- SQL Injection: SQL injection vulnerabilities allow one to manipulate, create or execute arbitrary SQL queries. This is the second most serious vulnerability on the OWASP Top 10 List, and the WackoPicko web application contains both a reflected and a stored SQL injection vulnerability.

- Code Injection: Code injection vulnerabilities allow an attacker to execute arbitrary commands or execute arbitrary code. This is the third most serious vulnerability on the OWASP Top 10 List, and WackoPicko includes both a command line injection and a file inclusion vulnerability (which might result in the execution of code).

- Broken Access Controls: A web application with broken access controls fails to properly define or enforce access to some of its resources. This is the tenth most serious vulnerability on the OWASP Top 10 List, and WackoPicko has an instance of this kind of vulnerability.

\subsection{Web Application Scanners}

In abstract, web application scanners can be seen as consisting of three main modules: a crawler module, an attacker module, and an analysis module. The crawling component is seeded with a set of URLs, retrieves the corresponding pages, and follows links and redirects to identify all the reachable pages in the application. In addition, the crawler identifies all the input points to the application, such as the parameters of GET requests, the input fields of HTML forms, and the controls that allow one to upload files.

The attacker module analyzes the URLs discovered by the crawler and the corresponding input points. Then, for each input and for each vulnerability type for which the web application vulnerability scanner tests, the attacker module generates values that are likely to trigger a vulnerability. For example, the attacker module would attempt to inject JavaScript code when testing for XSS vulnerabilities, or strings that have a special meaning in the SQL language, such as ticks and SQL operators, when testing 
for SQL injection vulnerabilities. Input values are usually generated using heuristics or using predefined values, such as those contained in one of the many available XSS and SQL injection cheat-sheets $[18,19]$.

The analysis module analyzes the pages returned by the web application in response to the attacks launched by the attacker module to detect possible vulnerabilities and to provide feedback to the other modules. For example, if the page returned in response to input testing for SQL injection contains a database error message, the analysis module may infer the existence of a SQL injection vulnerability.

\section{The WackoPicko Web Site}

A preliminary step for assessing web application scanners consists of choosing a web application to be tested. We have three requirements for such an application: it must have clearly defined vulnerabilities (to assess the scanner's detection performance), it must be easily customizable (to add crawling challenges and experiment with different types of vulnerabilities), and it must be representative of the web applications in use today (in terms of functionality and of technologies used).

We found that existing applications did not satisfy our requirements. Applications that deliberately contain vulnerabilities, such as HacmeBank [5] and WebGoat [11], are often designed to be educational tools rather than realistic testbeds for scanners. Others, such as SiteGenerator [10], are well-known, and certain scanners may be optimized to perform well on them. An alternative then is to use an older version of an open-source application that has known vulnerabilities. In this case, however, we would not be able to control and test the crawling capabilities of the scanners, and there would be no way to establish a false negative rate.

Therefore, we decided to create our own test application, called WackoPicko. It is important to note that WackoPicko is a realistic, fully functional web application. As opposed to a simple test application that contains just vulnerabilities, WackoPicko tests the scanners under realistic conditions. To test the scanners' support for clientside JavaScript code, we also used the open source Web Input Vector Extractor Teaser (WIVET). WIVET is a synthetic benchmark that measures how well a crawler is able to discover and follow links in a variety of formats, such as JavaScript, Flash, and form submissions.

\subsection{Design}

WackoPicko is a photo sharing and photo-purchasing site. A typical user of WackoPicko is able to upload photos, browse other user's photos, comment on photos, and purchase the rights to a high-quality version of a photo.

Authentication. WackoPicko provides personalized content to registered users. Despite recent efforts for a unified login across web sites [14], most web applications require a user to create an account in order to utilize the services offered. Thus, WackoPicko has a user registration system. Once a user has created an account, he/she can log in to access WackoPicko's restricted features.

Upload Pictures. When a photo is uploaded to WackoPicko by a registered user, other users can comment on it, as well as purchase the right to a high-quality version. 
Comment On Pictures. Once a picture is uploaded into WackoPicko, all registered users can comment on the photo by filling out a form. Once created, the comment is displayed, along with the picture, with all the other comments associated with the picture.

Purchase Pictures. A registered user on WackoPicko can purchase the high-quality version of a picture. The purchase follows a multi-step process in which a shopping cart is filled with the items to be purchased, similar to the process used in e-commerce sites. After pictures are added to the cart, the total price of the cart is reviewed, discount coupons may be applied, and the order is placed. Once the pictures are purchased, the user is provided with links to the high-quality version of the pictures.

Search. To enable users to easily search for various pictures, WackoPicko provides a search toolbar at the top of every page. The search functionality utilizes the tag field that was filled out when the picture was uploaded. After a query is issued, the user is presented with a list of all the pictures that have tags that match the query.

Guestbook. A guestbook page provides a way to receive feedback from all visitors to the WackoPicko web site. The form used to submit feedback contains a "name" field and a "comment" field.

Admin Area. WackoPicko has a special area for administrators only, which has a different login mechanism than regular users. Administrators can perform special actions, such as deleting user accounts, or changing the tags of a picture.

\subsection{Vulnerabilities}

The WackoPicko web site contains sixteen vulnerabilities that are representative of vulnerabilities found in the wild, as reported by the OWASP Top 10 Project [13]. In the following we provide a brief description of each vulnerability.

3.2.1 Publicly Accessible Vulnerabilities A number of vulnerabilities in WackoPicko can be exploited without first logging into the web site.

Reflected XSS: There is a XSS vulnerability on the search page, which is accessible without having to log into the application. In fact, the query parameter is not sanitized before being echoed to the user. The presence of the vulnerability can be tested by setting the query parameter to <script>alert $\left({ }^{\prime} x S^{\prime}{ }^{\prime}\right)</$ script $>$. When this string is reflected to the user, it will cause the browser to display an alert message. (Of course, an attacker would leverage the vulnerability to perform some malicious activity rather than alerting the victim.)

Stored XSS: There is a stored XSS vulnerability in the guestbook page. The comment field is not properly escaped, and therefore, an attacker can exploit this vulnerability by creating a comment containing JavaScript code. Whenever a user visits the guestbook page, the attack will be triggered and the (possibly malicious) JavaScript code executed. Session ID: The session information associated with administrative accounts is handled differently than the information associated with the sessions of normal users. The functionality associated with normal users uses PHP's session handling capabilities, which is assumed to be free of any session-related vulnerabilities (e.g., session fixation, easily-guessable session IDs). However the admin section uses a custom session cookie 
to keep track of sessions. The value used in the cookie is a non-random value that is incremented when a new session is created. Therefore, an attacker can easily guess the session id and access the application with administrative rights.

Weak password: The administrative account page has an easily-guessable username and password combination: admin/admin.

Reflected SQL Injection: WackoPicko contains a reflected SQL injection in the username field of the login form. By introducing a tick into the username field it is possible to perform arbitrary queries in the database and obtain, for example, the usernames and passwords of all the users in the system.

Command Line Injection: WackoPicko provides a simple service that checks to see if a user's password can be found in the dictionary. The password parameter of the form used to request the check is used without sanitization in the shell command: $g r e p$ <password>\$ / etc/dictionaries-common/words. This can be exploited by providing as the password value a dollar sign (to close grep's regular expression), followed by a semicolon (to terminate the grep command), followed by extra commands.

File Inclusion: The admin interface is accessed through a main page, called index.php. The index page acts as a portal; any value that is passed as its page parameter will be concatenated with the string ".php", and then the resulting PHP script will be run. For instance, the URL for the admin login page is / admin/index.php?page=login. On the server side, index.php will execute login.php which displays the form. This design is inherently flawed, because it introduces a file inclusion vulnerability. An attacker can exploit this vulnerability and execute remote PHP code by supplying, for example, http://hacker/blah.php 00 as the page parameter to index.php. The $\div 00$ at the end of the string causes PHP to ignore the ".php" that is appended to the page parameter. Thus index.php will download and execute the code at http: //hacker/blah.php.

Unauthorized File Exposure: In addition to executing remote code, the file inclusion vulnerability can also be exploited to expose local files. Passing / etc/passwdo 00 as the "page" GET parameter to index.php of the admin section will cause the contents of the /etc/passwd file to be disclosed.

Reflected XSS Behind JavaScript: On WackoPicko's home page there is a form that checks if a file is in the proper format for WackoPicko to process. This form has two parameters, a file parameter and a name parameter. Upon a successful upload, the name is echoed back to the user unsanitized, and therefore, this represents a reflected vulnerability. However, the form is dynamically generated using JavaScript, and the target of the form is dynamically created by concatenating strings. This prevents a crawler from using simple pattern matching to discover the URL used by the form.

Parameter Manipulation: The WackoPicko home page provides a link to a sample profile page. The link uses the "userid" GET parameter to view the sample user (who has id of 1). An attacker can manipulate this variable to view any profile page without having a valid user account.

3.2.2 Vulnerabilities Requiring Authentication A second class of vulnerabilities in WackoPicko can be exploited only after logging into the web site. 
Stored SQL Injection: When users create an account, they are asked to supply their first name. This supplied value is then used unsanitized on a page that shows other users who have a similar first name. An attacker can exploit this vulnerability by creating a user with the name "' ; DROP users;\#" then visiting the similar users page.

Directory Traversal: When uploading a picture, WackoPicko copies the file uploaded by the user to a subdirectory of the upload directory. The name of the subdirectory is the user-supplied tag of the uploaded picture. A malicious user can manipulate the tag parameter to perform a directory traversal attack. More precisely, by pre-pending "../. ./" to the tag parameter the attacker can reference files outside the upload directory and overwrite them.

Multi-Step Stored XSS: Similar to the stored XSS attack that exists on the guestbook, comments on pictures are susceptible to a stored XSS attack. However, this vulnerability is more difficult to exploit because the user must be logged in and must confirm the preview of the comment before the attack is actually triggered.

Forceful Browsing: One of the central ideas behind WackoPicko is the ability of users to purchase the rights to high-quality versions of pictures. However, the access to the links to the high-quality version of the picture is not checked, and an attacker who acquires the URL of a high-quality picture can access it without creating an account, thus bypassing the authentication logic.

Logic Flaw: The coupon system suffers from a logic flaw, as a coupon can be applied multiple times to the same order reducing the final price of an order to zero.

Reflected XSS Behind Flash: On the user's home page there is a Flash form that asks the user for his/her favorite color. The resulting page is vulnerable to a reflected XSS attack, where the "value" parameter is echoed back to the user without being sanitized.

\subsection{Crawling Challenges}

Crawling is arguably the most important part of a web application vulnerability scanner; if the scanner's attack engine is poor, it might miss a vulnerability, but if its crawling engine is poor and cannot reach the vulnerability, then it will surely miss the vulnerability. Because of the critical nature of crawling, we have included several types of crawling challenges in WackoPicko, some of which hide vulnerabilities.

HTML Parsing. Malformed HTML makes it difficult for web application scanners to crawl web sites. For instance, a crawler must be able to navigate HTML frames and be able to upload a file. Even though these tasks are straightforward for a human user with a regular browser, they represent a challenge for crawlers.

Multi-Step Process. Even though most web sites are built on top of the stateless HTTP protocol, a variety of techniques are utilized to introduce state into web applications. In order to properly analyze a web site, web application vulnerability scanners must be able to understand the state-based transactions that take place. In WackoPicko, there are several state-based interactions.

Infinite Web Site. It is often the case that some dynamically-generated content will create a very large (possibly infinite) crawling space. For example, WackoPicko has the ability to display a daily calendar. Each page of the calendar displays the agenda for a given day and links to the page for the following day. A crawler that naively followed the links in the WackoPicko's calendar would end up trying to visit an infinite sequence of pages, all generated dynamically by the same component. 


\begin{tabular}{|l|l|r|r|r|}
\hline Name & Version Used & License & Type & Price \\
\hline Acunetix & 6.1 Build 20090318 & Commercial & Standalone & $\$ 4,995-\$ 6,350$ \\
AppScan & 7.8 .0 .0 iFix001 Build: 570 Security & Commercial & Standalone & $\$ 17,550-\$ 32,500$ \\
& Rules Version 647 & & \\
Burp & 1.2 & Commercial & Proxy & $£ 125(\$ 190.82)$ \\
Grendel-Scan & 1.0 & GPLv3 & Standalone & N/A \\
Hailstorm & 5.7 Build 3926 & Commercial & Standalone & $\$ 10,000$ \\
Milescan & 1.4 & Commercial & Proxy & $\$ 495-\$ 1,495$ \\
N-Stalker & $2009-$ Build 7.0.0.207 & Commercial & Standalone & $\$ 899-\$ 6,299$ \\
NTOSpider & 3.2 .067 & Commercial & Standalone & $\$ 10,000$ \\
Paros & 3.2 .13 & Clarified Artistic License & Proxy & N/A \\
w3af & $1.0-$ rc2 & GPLv2 & Standalone & N/A \\
Webinspect & 7.7 .869 .0 & Commercial & Standalone & $\$ 6,000-\$ 30,000$ \\
\hline
\end{tabular}

Table 1: Characteristics of the scanners evaluated.

Authentication. One feature that is common to most web sites is an authentication mechanism. Because this is so prevalent, scanners must properly handle authentication, possibly by creating accounts, logging in with valid credentials, and recognizing actions that log the crawler out. WackoPicko includes a registration and login system to test the scanner's crawlers ability to handle the authentication process correctly.

Client-side Code. Being able to parse and understand client-side technologies presents a major challenge for web application vulnerability scanners. WackoPicko includes vulnerabilities behind a JavaScript-created form, as well as behind a Flash application.

Link Extraction. We also tested the scanners on WIVET, an open-source benchmark for web link extractors [12]. WIVET contains 54 tests and assigns a final score to a crawler based on the percent of tests that it passes. The tests require scanners to analyze simple links, multi-page forms, links in comments and JavaScript actions on a variety of HTML elements. There are also AJAX-based tests as well as Flash-based tests. In our tests, we used WIVET version number 129.

\section{Experimental Evaluation}

We tested 11 web application scanners by running them on our WackoPicko web site. The tested scanners included 8 proprietary tools and 3 open source programs. Their cost ranges from free to tens of thousands of dollars. We used evaluation versions of each software, however they were fully functional. A summary of the characteristics of the scanners we evaluated is given in Table 1 .

We ran the WackoPicko web application on a typical LAMP machine, with Apache 2.2.9, PHP 5.2.6, and MySQL 5.0.67. We enabled the al low_ur l_fopen and al low_url_include PHP options and disabled the magic_quotes option. We ran the scanners on a machine with a Pentium $43.6 \mathrm{GHz}$ CPU, $1024 \mathrm{MB}$ of RAM, and Microsoft Windows XP, Service Pack 2.

\subsection{Setup}

The WackoPicko server used in testing the web vulnerability scanners was run in a virtual machine, so that before each test run the server could be put in an identical initial state. This state included ten regular users, nine pictures, and five administrator users. 


\begin{tabular}{|c|c|c|c|c|c|c|c|c|}
\hline Name & $\begin{array}{l}\text { Reflected } \\
\text { XSS }\end{array}$ & Stored XSS & \begin{tabular}{|l|} 
Reflected \\
SQL \\
Injection
\end{tabular} & $\begin{array}{l}\text { Command- } \\
\text { line } \\
\text { Injection }\end{array}$ & \begin{tabular}{|l} 
File \\
Inclusion
\end{tabular} & \begin{tabular}{|l} 
File \\
Exposure
\end{tabular} & \begin{tabular}{|l} 
XSS via \\
JavaScript
\end{tabular} & $\begin{array}{l}\text { XSS via } \\
\text { Flash }\end{array}$ \\
\hline Acunetix & INITIAL & INITIAL & INITIAL & \multirow{11}{*}{ INITIAL } & \multirow{11}{*}{\begin{tabular}{|l} 
INITIAL \\
INITIAL
\end{tabular}} & \multirow{11}{*}{$\begin{array}{l}\text { INITIAL } \\
\text { INITIAL } \\
\text { INITIAL }\end{array}$} & INITIAL & \multirow{11}{*}{$\begin{array}{l}\text { MANUAL } \\
\text { MANUAL } \\
\text { MANUAL } \\
\text { MANUAL } \\
\text { MANUAL } \\
\text { MANUAL }\end{array}$} \\
\hline AppScan & INITIAL & INITIAL & INITIAL & & & & & \\
\hline Burp & INITIAL & MANUAL & INITIAL & & & & & \\
\hline Grendel-Scan & MANUAL & & CONFIG & & & & & \\
\hline Hailstorm & INITIAL & CONFIG & CONFIG & & & & & \\
\hline Milescan & INITIAL & MANUAL & CONFIG & & & & & \\
\hline N-Stalker & INITIAL & MANUAL & MANUAL & & & & INITIAL & \\
\hline NTOSpider & INITIAL & INITIAL & INITIAL & & & & & \\
\hline Paros & INITIAL & INITIAL & CONFIG & & & & & \\
\hline w3af & INITIAL & MANUAL & INITIAL & & & & & \\
\hline Webinspect & INITIAL & INITIAL & INITIAL & & & & INITIAL & \\
\hline
\end{tabular}

Table 2: Detection results. For each scanner, the simplest configuration that detected a vulnerability is given. Empty cells indicate no detection in any mode.

Each scanner was run in three different configuration modes against WackoPicko, with each configuration requiring more setup on the part of the user. In all configuration styles, the default values for configuration parameters were used, and when choices were required, sensible values were chosen. In the INITIAL configuration mode, the scanner was directed to the initial page of WackoPicko and told to scan for all vulnerabilities. In the CONFIG setup, the scanner was given a valid username/password combination or login macro before scanning. MANUAL configuration required the most work on the part of the user; each scanner was put into a "proxy" mode and then the user browsed to each vulnerable page accessible without credentials; then, the user logged in and visited each vulnerability that required a login. Additionally a picture was uploaded, the rights to a high-quality version of a picture were purchased, and a coupon was applied to the order. The scanner was then asked to scan the WackoPicko web site.

\subsection{Detection Results}

The results of running the scanners against the WackoPicko site are shown in Table 2 and, graphically, in Figure 1. The values in the table correspond to the simplest configuration that discovered the vulnerability. An empty cell indicates that the given scanner did not discover the vulnerability in any mode. The table only reports the vulnerabilities that were detected by at least one scanner. Further analysis of why the scanners missed certain vulnerabilities is contained in Sections 4.3 and 4.4.

The running time of the scanners is shown in Figure 3. These times range from 74 seconds for the fastest tool (Burp) to 6 hours (N-Stalker). The majority of the scanners completed the scan within a half hour, which is acceptable for an automated tool.

4.2.1 False Negatives One of the benefits of developing the WackoPicko web application to test the scanners is the ability for us to measure the false negatives of the scanners. An ideal scanner would be able to detect all vulnerabilities. In fact, we had a group composed of students with average security skills analyze WackoPicko. The students found all vulnerabilities except for the forceful browsing vulnerability. The automated scanners did not do as well; there were a number of vulnerabilities that were not detected by any scanner. These vulnerabilities are discussed hereinafter. 
Session ID: No scanner was able to detect the session ID vulnerability on the admin login page. The vulnerability was not detected because the scanners were not given a valid username/password combination for the admin interface. This is consistent with what would happen when scanning a typical application, as the administration interface would include powerful functionality that the scanner should not invoke, like view, create, edit or delete sensitive user data. The session ID was only set on a successful login, which is why this vulnerability was not detected by any scanner.

Weak Password: Even though the scanners were not given a valid username/password combination for the administrator web site, an administrator account with the combination of admin/admin was present on the system. NTOSpider was the only scanner that successfully logged in with the admin/admin combination. However, it did not report it as an error, which suggests that it was unable to detect that the login was successful, even though the response that was returned for this request was different from every other login attempt.

Parameter Manipulation: The parameter manipulation vulnerability was not discovered by any scanner. There were two causes for this: first, only three of the scanners (AppScan, NTOSpider, and w3af) input a different number than the default value "1" to the userid parameter. Of the three, only NTOSpider used a value that successfully manipulated the userid parameter. The other reason was that in order to successfully detect a parameter manipulation vulnerability, the scanner needs to determine which pages require a valid username/password to access and which ones do not and it is clear that none of the scanners make this determination.

Stored SQL Injection: The stored SQL injection was also not discovered by any scanners, due to the fact that a scanner must create an account to discover the stored SQL injection. The reasons for this are discussed in more detail in Section 4.4.4.

Directory Traversal: The directory traversal vulnerability was also not discovered by any of the scanners. This failure is caused by the scanners being unable to upload a picture. We discuss this issue in Section 4.4.2, when we analyze how each of the scanners behaved when they had to upload a picture.

Multi-Step Stored XSS: The stored XSS vulnerability that required a confirmation step was also missed by every scanner. In Section 4.4.5, we analyze how many of the scanners were able to successfully create a comment on a picture.

Forceful Browsing: No scanner found the forceful browsing vulnerability, which is not surprising since it is an application-specific vulnerability. These vulnerabilities are difficult to identify without access to the source code of the application [2].

Logic Flaw: Another vulnerability that none of the scanners uncovered was the logic flaw that existed in the coupon management functionality. Also in this case, some domain knowledge about the application is needed to find the vulnerability. 


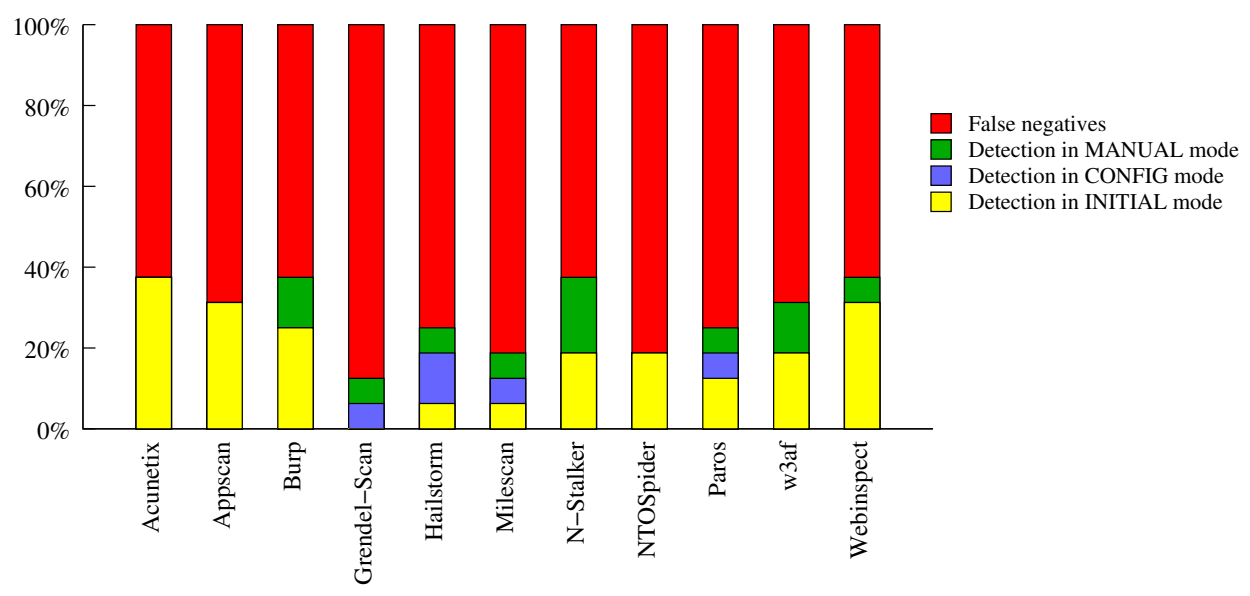

Fig. 1: Detection performance (true positives and false negatives) of the evaluated scanners.

\begin{tabular}{|l|r|r|r|}
\hline Name & INITIAL & CONFIG & MANUAL \\
\hline Acunetix & 1 & 7 & 4 \\
AppScan & 11 & 20 & 26 \\
Burp & 1 & 2 & 6 \\
Grendel-Scan & 15 & 16 & 16 \\
Hailstorm & 3 & 11 & 3 \\
Milescan & 0 & 0 & 0 \\
N-Stalker & 5 & 0 & 0 \\
NTOSpider & 3 & 1 & 3 \\
Paros & 1 & 1 & 1 \\
w3af & 1 & 1 & 9 \\
Webinspect & 215 & 317 & 297 \\
\hline
\end{tabular}

Table 3: False positives.

4.2.2 False Positives The total number of false positives for each of the scanning configurations are show in Table 3 . The number of false positives that each scanner generates is an important metric, because the greater the number of false positives, the less useful the tool is to the end user, who has to figure out which of the vulnerabilities reported are actual flaws and which are spurious results of the analysis.

The majority of the false positives across all scanners were due to a supposed "Server Path Disclosure." This is an information leakage vulnerability where the server leaks the paths of local files, which might give an attacker hints about the structure of the file system.

An analysis of the results identified two main reasons why these false positives were generated. The first is that while testing the application for file traversal or file injection vulnerabilities, some of the scanners passed parameters with values of file names, which, on some pages (e.g., the guestbook page), caused the file name to be included in that page's contents. When the scanner then tested the page for a Server Path Disclosure, it found the injected values in the page content, and generated a Server Path Disclosure vulnerability report. The other reason for the generation of false positives is that WackoPicko uses absolute paths in the href attribute of anchors (e.g., / users/home.php), which the scanner mistook for the disclosure of paths in the 


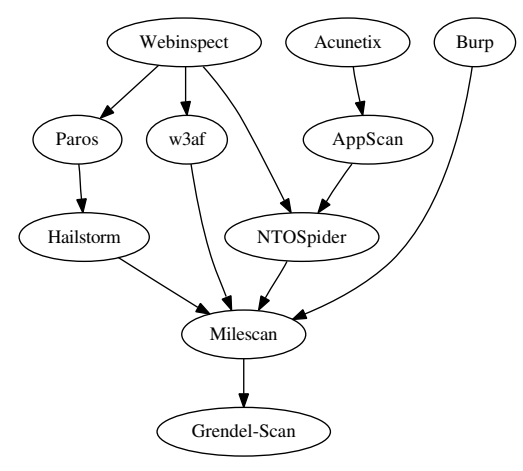

Fig. 2: Dominates graph.

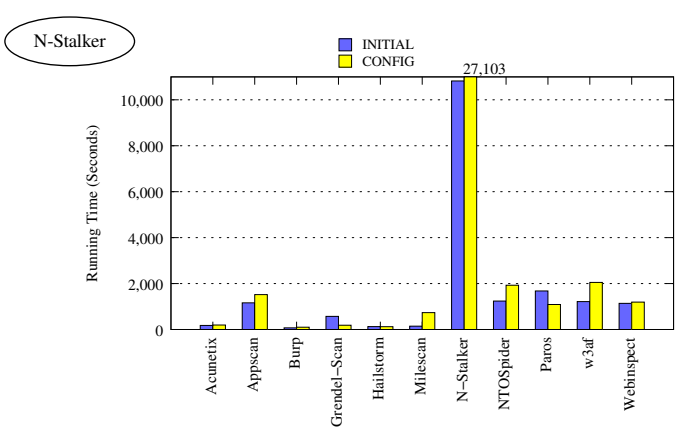

Fig. 3: Scanner Running Times

local system. Webinspect generated false positives because of both the above reasons, which explains the large amount of false positives produced by the tool.

Some scanners reported genuine false positives: Hailstorm reported a false XSS vulnerability and two false PHP code injection vulnerabilities, NTOSpider reported three false XSS vulnerabilities and w3af reported a false PHP eval ( ) input injection vulnerability.

4.2.3 Measuring and Comparing Detection Capabilities Comparing the scanners using a single benchmark like WackoPicko does not represent an exhaustive evaluation. However, we believe that the results provide insights about the current state of blackbox web application vulnerability scanners.

One possible way of comparing the results of the scanners is arranging them in a lattice. This lattice is ordered on the basis of strict dominance. Scanner A strictly dominates Scanner B if and only if for every vulnerability discovered by Scanner B, Scanner A discovered that vulnerability with the same configuration level or simpler, and Scanner A either discovered a vulnerability that Scanner B did not discover or Scanner A discovered a vulnerability that Scanner B discovered, but with a simpler configuration. Strictly dominates has the property that any assignment of scores to vulnerabilities must preserve the strictly dominates relationship.

Figure 2 shows the strictly dominates graph for the scanners, where a directed edge from Scanner A to Scanner B means that Scanner A strictly dominates Scanner B. Because strictly dominates is transitive, if one scanner strictly dominates another it also strictly dominates all the scanners that the dominated scanner dominates, therefore, all redundant edges are not included. Figure 2 is organized so that the scanners in the top level are those that are not strictly dominated by any scanners. Those in the second level are strictly dominated by only one scanner and so on, until the last level, which contains those scanners that strictly dominate no other scanner.

Some interesting observations arise from Figure 2. N-Stalker does not strictly dominate any scanner and no scanner strictly dominates it. This is due to the unique combination of vulnerabilities that N-Stalker discovered and missed. Burp is also interesting 


\begin{tabular}{|l|l|l|l|l|}
\hline Name & Detection & $\begin{array}{l}\text { INITIAL } \\
\text { Reachability }\end{array}$ & $\begin{array}{l}\text { CONFIG } \\
\text { Reachability }\end{array}$ & $\begin{array}{l}\text { MANUAL } \\
\text { Reachability }\end{array}$ \\
\hline XSS Reflected & 1 & 0 & 0 & 0 \\
XSS Stored & 2 & 0 & 0 & 0 \\
SessionID & 4 & 0 & 0 & 0 \\
SQL Injection Reflected & 1 & 0 & 0 & 0 \\
Commandline Injection & 4 & 0 & 0 & 0 \\
File Inclusion & 3 & 0 & 0 & 0 \\
File Exposure & 3 & 0 & 0 & 0 \\
XSS Reflected behind & 1 & 3 & 3 & 0 \\
JavaScript & 8 & 0 & 0 \\
Parameter Manipulation & 8 & 0 & 0 & 0 \\
Weak password & 3 & 0 & 3 & 3 \\
SQL Injection Stored Login & 7 & 7 & 6 & 4 \\
Directory Traversal Login & 8 & 8 & 6 \\
XSS Stored Login & 2 & 8 & 6 & 3 \\
Forceful Browsing Login & 8 & 7 & 8 & 6 \\
Logic Flaws - Coupon & 9 & 9 & 7 & 1 \\
XSS Reflected behind flash & 1 & 9 & \\
\hline
\end{tabular}

\begin{tabular}{|l|r|}
\hline Name & Score \\
\hline Acunetix & 14 \\
Webinspect & 13 \\
Burp & 13 \\
N-Stalker & 13 \\
AppScan & 10 \\
w3af & 9 \\
Paros & 6 \\
Hailstorm & 6 \\
NTOSpider & 4 \\
Milescan & 4 \\
Grendel-Scan & 3 \\
\hline
\end{tabular}

Table 5: Final ranking.

Table 4: Vulnerability scores.

due to the fact that it only dominates two scanners but no scanner dominates Burp because it was the only scanner to discover the command-line injection vulnerability.

While Figure 2 is interesting, it does not give a way to compare two scanners where one does not strictly dominate the other. In order to compare the scanners, we assigned scores to each vulnerability present in WackoPicko. The scores are displayed in Table 4. The "Detection" score column in Table 4 is how many points a scanner is awarded based on how difficult it is for an automated tool to detect the existence of the vulnerability. In addition to the "Detection" score, each vulnerability is assigned a "Reachability" score, which indicates how difficult the vulnerability is to reach (i.e., it reflects the difficulty of crawling to the page that contains the vulnerability). There are three "Reachability" scores for each vulnerability, corresponding to how difficult it is for a scanner to reach the vulnerability when run in INITIAL, CONFIG, or MANUAL mode. Of course, these vulnerability scores are subjective and depend on the specific characteristics of our WackoPicko application. However, their values try to estimate the crawling and detection difficulty of each vulnerability in this context.

The final score for each scanner is calculated by adding up the "Detection" score for each vulnerability the scanner detected and the "Reachability" score for the configuration (INITIAL, CONFIG and MANUAL) used when running the scanner. In the case of a tie, the scanners were ranked by how many vulnerabilities were discovered in INITIAL mode, which was enough to break all ties. Table 5 shows the final ranking of the scanners.

\subsection{Attack and Analysis Capabilities}

Analyzing how each scanner attempted to detect vulnerabilities gives us insight into how these programs work and illuminates areas for further research. First, the scanner would crawl the site looking for injection points, typically in the form of GET or POST parameters. Once the scanner identifies all the inputs on a page, it then attempts to inject values for each parameter and observes the response. When a page has more than one input, each parameter is injected in turn, and generally no two parameters are 
injected in the same request. However, scanners differ in what they supply as values of the non-injected parameters: some have a default value like 1234 or Peter Wiener, while others leave the fields blank. This has an impact on the results of the scanner, for example the WackoPicko guestbook requires that both the name and comment fields are present before making a comment, and thus the strategy employed by each scanner can affect the effectiveness of the vulnerability scanning process.

When detecting XSS attacks, most scanners employed similar techniques, some with a more sophisticated attempt to evade possible filters than others. One particularly effective strategy employed was to first input random data with various combinations of dangerous characters, such as $/, ",{ }^{\prime},<$, and $>$, and then, if one of these combinations was found unchanged in the response, to attempt the injection of the full range of XSS attacks. This technique speeds up the analysis significantly, because the full XSS attack is not attempted against every input vector. Differently, some of the scanners took an exhaustive approach, attempting the full gamut of attacks on every combination of inputs.

When attempting a XSS attack, the thorough scanners would inject the typical <script> alert ('XSS') </script> as well as a whole range of XSS attack strings, such as JavaScript in a tag with the onmouseover attribute, in an img, div or meta tag, or iframe. Other scanners attempted to evade filters by using a different JavaScript function other than a lert, or by using a different casing of script, such as ScRiPt.

Unlike with XSS, scanners could not perform an easy test to exclude a parameter from thorough testing for other Unsanitized Input vulnerabilities because the results of a successful exploit might not be readily evident in the response. This is true for the command-line injection on the WackoPicko site, because the output of the injectable command was not used in the response. Burp, the only scanner that was able to successfully detect the command line injection vulnerability, did so by injecting "ping $-\mathrm{C} 100$ localhost' and noticing that the response time for the page was much slower than when nothing was injected.

This pattern of measuring the difference in response times was also seen in detecting SQL injections. In addition to injecting something with a SQL control character, such as tick or quote and seeing if an error is generated, the scanners also used a time-delay SQL injection, inputting waitfor delay ${ }^{\prime} 0: 0: 20^{\prime}$ and seeing if the execution was delayed. This is a variation of the technique of using time-delay SQL injection to extract database information from a blind SQL vulnerability.

When testing for File Exposure, the scanners were typically the same; however one aspect caused them to miss the WackoPicko vulnerability. Each scanner that was looking for this vulnerability input the name of a file that they knew existed on the system, such as /etc/passwd on UNIX-like systems or C: \boot. ini for Windows. The scanners then looked for known strings in the response. The difficulty in exploiting the WackoPicko file exposure was including the null-terminating character $(\% 00)$ at the end of the string, which caused PHP to ignore anything added by the application after the / etc/passwd part. The results show that only 4 scanners successfully discovered this vulnerability.

The remote code execution vulnerability in WackoPicko is similar to the file exposure vulnerability. However, instead of injecting known files, the scanners injected 


\begin{tabular}{|c|c|c|c|c|c|c|c|c|c|c|c|c|c|c|c|c|c|c|}
\hline Name & \multicolumn{3}{|c|}{ Reflected XSS } & \multicolumn{3}{|c|}{ Stored XSS } & \multicolumn{3}{|c|}{$\begin{array}{l}\text { Reflected SQL } \\
\text { Injection }\end{array}$} & \multicolumn{3}{|c|}{$\begin{array}{l}\text { Command-line } \\
\text { Injection }\end{array}$} & \multicolumn{3}{|c|}{$\begin{array}{l}\text { File Inclusion / } \\
\text { File Exposure / } \\
\text { Weak password }\end{array}$} & \multicolumn{3}{|c|}{$\begin{array}{l}\text { XSS Reflected } \\
\text { - JavaScript }\end{array}$} \\
\hline Acunetix & 496 & 638 & 498 & 613 & 779 & 724 & 544 & 709 & 546 & 495 & 637 & 497 & 198 & 244 & 200 & 670 & 860 & 671 \\
\hline AppScan & 581 & 575 & 817 & 381 & 352 & 492 & 274 & 933 & 628 & 189 & 191 & 288 & 267 & 258 & 430 & & 0 & 442 \\
\hline Burp & 2 & 256 & 207 & 192 & 192 & 262 & 68 & 222 & 221 & 68 & 68 & 200 & 125 & 316 & 320 & & 0 & 178 \\
\hline Grendel-Scan & 0 & 0 & 44 & 1 & 1 & 3 & 14 & 34 & 44 & 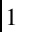 & 1 & 3 & 2 & 2 & 5 & & 0 & 2 \\
\hline Hails & 232 & 229 & 233 & 10 & 205 & 209 & 45 & 224 & 231 & 180 & 160 & 162 & 8 & 204 & 216 & 153 & 147 & 148 \\
\hline Miles & 104 & 0 & 208 & 50 & 0 & 170 & 75 & 272 & 1237 & 0 & 0 & 131 & 80 & 0 & 246 & & 0 & 163 \\
\hline N-Stal & 1738 & 1162 & 2689 & 2484 & 2100 & 3475 & 2764 & +1022 & 2110 & 200 & 5189 & 1987 & 1437 & 72063 & 31824 & 1409 & 1292 & 1335 \\
\hline NTOSpider & 856 & 679 & 692 & 252 & 370 & 370 & 184 & 5 & 5 & 105 & 9 & 9 & 243 & 614 & 614 & 11 & 13 & 13 \\
\hline Paros & 68 & 68 & 58 & 126 & 126 & 110 & 151 & 299 & 97 & 28 & 28 & 72 & 146 & 146 & 185 & & 0 & 56 \\
\hline w3af & 157 & 157 & 563 & 259 & 257 & 464 & 1377 & 71411 & 2634 & 140 & 142 & 253 & 263 & 262 & 470 & & 0 & 34 \\
\hline Webinspect & 108 & 108 & 105 & 631 & 631 & 630 & 297 & 403 & 346 & 164 & 164 & 164 & 239 & 237 & 234 & 909 & 909 & 0 \\
\hline Name & \multicolumn{3}{|c|}{ Parameter Manipulation } & \multicolumn{3}{|c|}{$\begin{array}{l}\text { Directory } \\
\text { Traversal }\end{array}$} & \multicolumn{3}{|c|}{ Logic Flaw } & \multicolumn{3}{|c|}{$\begin{array}{l}\text { Forceful } \\
\text { Browsing }\end{array}$} & \multicolumn{3}{|c|}{$\begin{array}{l}\text { XSS Reflected } \\
\text { behind flash }\end{array}$} & & & \\
\hline Acunetix & 2 & 0 & 2 & 35 & 1149 & 937 & 0 & 0 & 5 & $0_{0}$ & 0 & 206 & 1 & 34 & 458 & & & \\
\hline AppScan & 221 & 210 & 222 & 80 & 70 & 941 & 0 & 0 & 329 & o & 0 & 71 & 0 & 0 & 243 & & & \\
\hline Burp & 192 & 194 & 124 & 68 & 68 & 394 & 0 & 0 & 314 & 0 & 0 & 151 & 0 & 0 & 125 & & & \\
\hline Grendel-Scan & 3 & 3 & 6 & 1 & 1 & 3 & 0 & 0 & 6 & 0 & 0 & 1 & 0 & 0 & 3 & & & \\
\hline Hailstorm & 3 & 143 & 146 & 336 & 329 & 344 & 131 & 132 & 5 & 102 & 102 & 105 & 0 & 0 & 143 & & & \\
\hline Milescan & 105 & 0 & 103 & 8 & 0 & 163 & 0 & 0 & 1 & 0 & 0 & 60 & 0 & 0 & 68 & & & \\
\hline $\mathrm{N}$-Stalker & 1291 & 1270 & 1302 & 22 & 2079 & 9704 & 0 & 0 & 3 & 0 & 0 & 2 & 0 & 0 & 1315 & & & \\
\hline NTOSpider & 107 & 115 & 115 & 11 & 572 & 572 & 0 & 11 & 11 & 0 & 0 & 0 & 0 & 11 & 11 & & & \\
\hline Paros & 72 & 72 & 72 & 14 & 14 & 0 & 0 & 0 & 114 & 0 & 0 & 70 & 0 & 0 & 60 & & & \\
\hline w3af & 128 & 128 & 124 & 31 & 30 & 783 & 0 & 0 & 235 & 0 & 0 & 270 & 0 & 0 & 119 & & & \\
\hline Webinspect & 102 & 102 & 102 & 29 & 29 & 690 & 0 & 8 & 3 & 0 & 118 & 82 & 0 & 0 & 97 & & & \\
\hline
\end{tabular}

Table 6: Number of accesses to vulnerable web pages in INITIAL, CONFIG, and MANUAL modes.

known web site addresses. This was typically from a domain the scanner's developers owned, and thus when successfully exploited, the injected page appeared instead of the regular page. The same difficulty in a successful exploitation existed in the File Exposure vulnerability, so a scanner had to add $\div 00$ after the injected web site. Only 3 scanners were able to successfully identify this vulnerability.

\subsection{Crawling Capabilities}

The number of URLs requested and accessed varies considerably among scanners, depending on the capability and strategies implemented in the crawler and attack components. Table 6 shows the number of times each scanner made a POST or GET request to a vulnerable URL when the scanners were run in INITIAL, CONFIG, and MANUAL mode. For instance, from Table 6 we can see that Hailstorm was able to access many of the vulnerable pages that required a valid username/password when run in INITIAL mode. It can also be seen that N-Stalker takes a shotgun-like approach to scanning; it has over 1,000 accesses for each vulnerable URL, while in contrast Grendel-Scan never had over 50 accesses to a vulnerable URL.

In the following, we discuss the main challenges that the crawler components of the web application scanners under test faced.

4.4.1 HTML The results for the stored XSS attack reveal some interesting characteristics of the analysis performed by the various scanners. For instance, Burp, GrendelScan, Hailstorm, Milescan, N-Stalker, and w3af were unable to discover the stored XSS 
vulnerability in INITIAL configuration mode. Burp and N-Stalker failed because of defective HTML parsing. Neither of the scanners correctly interpreted the <textarea> tag as an input to the HTML form. This was evident because both scanners only sent the name parameter when attempting to leave a comment on the guestbook. When run in MANUAL mode, however, the scanners discovered the vulnerability, because the user provided values for all these fields. Grendel-Scan and Milescan missed the stored XSS vulnerability for the same reason: they did not attempt a POST request unless the user used the proxy to make the request.

Hailstorm did not try to inject any values to the guestbook when in INITIAL mode, and, instead, used testval as the name parameter and Default text as the comment parameter. One explanation for this could be that Hailstorm was run in the default "turbo" mode, which Cenzic claims catches $95 \%$ of vulnerabilities, and chose not to fuzz the form to improve speed.

Finally, w3af missed the stored XSS vulnerability due to leaving one parameter blank while attempting to inject the other parameter. It was unable to create a guestbook entry, because both parameters are required.

4.4.2 Uploading a Picture Being able to upload a picture is critical to discover the Directory Traversal vulnerability, as a properly crafted tag parameter can overwrite any file the web server can access. It was very difficult for the scanners to successfully upload a file: no scanner was able to upload a picture in INITIAL and CONFIG modes, and only AppScan and Webinspect were able to upload a picture after being showed how to do it in MANUAL configuration, with AppScan and Webinspect uploading 324 and 166 pictures respectively. Interestingly, Hailstorm, N-Stalker and NTOSpider never successfully uploaded a picture, even in MANUAL configuration. This surprising result is due to poor proxies or poor in-application browsers. For instance, Hailstorm includes an embedded Mozilla browser for the user to browse the site when they want to do so manually, and after repeated attempts the embedded browser was never able to upload a file. The other scanners that failed, N-Stalker and NTOSpider, had faulty HTTP proxies that did not know how to properly forward the file uploaded, thus the request never completed successfully.

4.4.3 Client-side Code The results of the WIVET tests are shown in Figure 4. Analyzing the WIVET results gives a very good idea of the JavaScript capabilities of each scanner. Of all the 54 WIVET tests, 24 required actually executing or understand JavaScript code; that is, the test could not be passed simply by using a regular expression to extract the links on the page. Webinspect was the only scanner able to complete all of the dynamic JavaScript challenges. Of the rest of the scanners, Acunetix and NTOSpider only missed one of the dynamic JavaScript tests. Even though Hailstorm missed 12 of the dynamic JavaScript tests, we believe that this is because of a bug in the JavaScript analysis engine and not a general limitation of the tool. In fact, Hailstorm was able to correctly handle JavaScript on the onmouseup and onclick parametrized functions. These tests were on parametrized onmous eout and onmous edown functions, but since Hailstorm was able to correctly handle the onmou seup and onclick parametrized functions, this can be considered a bug in Hailstorm's JavaScript parsing. From this, it can also be concluded that AppScan, Grendel-Scan, Milescan, and w3af 
perform no dynamic JavaScript parsing. Thus, Webinspect, Acunetix, NTOSpider, and Hailstorm can be claimed to have the best JavaScript parsing. The fact that N-Stalker found the reflected XSS vulnerability behind a JavaScript form in WackoPicko suggests that it can execute JavaScript, however it failed the WIVET benchmark so we cannot evaluate the extent of the parsing performed.

In looking at the WIVET results, there was one benchmark that no scanner was able to reach, which was behind a Flash application. The application had a link on a button's onclick event, however this link was dynamically created at run time. This failure shows that none of the current scanners processes Flash content with the same level of sophistication as JavaScript. This conclusion is supported by none of the scanners discovering the XSS vulnerability behind a Flash application in WackoPicko when in

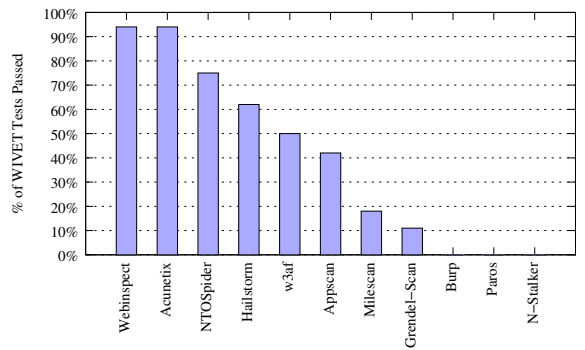

Fig. 4: WIVET results. INITIAL or CONFIG mode.

4.4.4 Authentication Table 7 shows the attempts that were made to create an account on the WackoPicko site. The Name column is the name of the scanner, "Successful" is the number of accounts successfully created, and "Error" is the number of account creation attempts that were unsuccessful. Note that Table 7 reports the results of the scanners when run in INITIAL mode only, because the results for the other configurations were almost identical.

Table 7 shows the capability of the scanners to handle user registration functionality. As can be seen from Table 7, only five of the scanners were able to successfully create an account. Of these, Hailstorm was the only one to leverage this ability to visit vulnerable URLs that required a login in its INITIAL run.

Creating an account is important in discovering the stored SQL injection that no scanner successfully detected. It is fairly telling that even though five scanners were able to create an account, none of them detected the vulnerability. It is entirely possible that none of the scanners actively searched for stored SQL injections, which is much harder to detect than stored XSS injections.

In addition to being critically important to the WackoPicko benchmark, being able to create an account is an important skill for a scanner to have when analyzing any web site, especially if that scanner wishes to be a point-and-click web application vulnerability scanner. 
4.4.5 Multi-step Processes In the WackoPicko web site there is a vulnerability that is triggered by going through a multi-step process. This vulnerability is the stored XSS on pictures, which requires an attacker to confirm a comment posting for the attack to be successful. Hailstorm and NTOSpider were the only scanners to successfully create a comment on the INITIAL run (creating 25 and 1 comment, respectively). This is important for two reasons: first, to be able to create a comment in the INITIAL run, the scanner had to create an account and $\log$ in with that account, which is consistent with Table 7. Also, all 25 of the comments successfully created by Hailstorm only contained the text Default text, which means that Hailstorm was not able to create a comment that exploited the vulnerability.

All scanners were able to create a comment when run in MANUAL configuration, since they were shown by the user how to carry out this task. However, only AppScan, Hailstorm, NTOSpider, and Webinspect (creating 6, 21, 7, and 2 comments respectively) were able to create a comment that was different than the one provided by the user. Of these scanners only Webinspect was able to create a comment that exploited the vulnerability, <iFrAmE sRc=hTtP://xSrFtEsT .sPi/> </iFrAmE>, however Webinspect failed to report this vulnerability. One plausible explanation for not detecting would be the scanners' XSS strategy discussed in Section 4.3. While testing the text parameter for a vulnerability, most of the scanners realized that it was properly escaped on the preview page, and thus stopped trying to inject XSS attacks. This would explain the directory traversal attack comment that AppScan successfully created and why Hailstorm did not attempt any injection. This is an example where the performance optimization of the vulnerability analysis can lead to false negatives.

4.4.6 Infinite Web Sites One of the scanners attempted to visit all of the pages of the infinite calendar. When running Grendel-Scan, the calendar portion of WackoPicko had to be removed because the scanner ran out of memory attempting to access every page. Acunetix, Burp, N-Stalker and w3af had the largest accesses (474, 691, 1780 and 3094 respectively), due to their attempts to exploit the calendar page. The other scanners used less accesses (between 27 and 243) because they were able to determine that no error was present.

\section{Lessons Learned}

We found that the crawling of modern web applications can be a serious challenge for today's web vulnerability scanners. A first class of problems we encountered consisted of implementation errors and the lack of support for commonly-used technologies. For example, handling of multimedia data (image uploads) exposed bugs in certain proxybased scanners, which prevented the tools from delivering attacks to the application under test. Incomplete or incorrect HTML parsers caused scanners to ignore input vectors that would have exposed vulnerabilities. The lack of support for JavaScript (and Flash) prevented tools from reaching vulnerable pages altogether. Support for wellknown, pervasive technology should be improved.

The second class of problems that hindered crawling is related to the design of modern web applications. In particular, applications with complex forms and aggressive checking of input values can effectively block a scanner, preventing it from crawling 
the pages "deep" in the web site structure. Handling this problem could be done, for example, by using heuristics to identify acceptable inputs or by reverse engineering the input filters. Furthermore, the behavior of an application can be wildly different depending on its internal "state," i.e., the values of internal variables that are not explicitly exposed to the scanner. The classic example of application state is whether the current user is logged in or not. A scanner that does not correctly model and track the state of an application (e.g., it does not realize that it has been automatically logged out) will fail to crawl all relevant parts of the application. More sophisticated algorithms are needed to perform "deep" crawling and track the state of the application under test.

Current scanners fail to detect (or even check for) application-specific (or "logic") vulnerabilities. Unfortunately, as applications become more complex, this type of vulnerabilities will also become more prevalent. More research is warranted to automate the detection of application logic vulnerabilities.

In conclusion, far from being point-and-click tools to be used by anybody, web application black-box security scanners require a sophisticated understanding of the application under test and of the limitations of the tool, in order to be effective.

\section{Related Work}

Our work is related to two main areas of research: the design of web applications for assessing vulnerability analysis tools and the evaluation of web scanners.

Designing test web applications. Vulnerable test applications are required to assess web vulnerability scanners. Unfortunately, no standard test suite is currently available or accepted by the industry. HacmeBank [5] and WebGoat [11] are two well-known, publicly-available, vulnerable web applications, but their design is focused more on teaching web application security rather than testing automated scanners. SiteGenerator [10] is a tool to generate sites with certain characteristics (e.g., classes of vulnerabilities) according to its input configuration. While SiteGenerator is useful to automatically produce different vulnerable sites, we found it easier to manually introduce in WackoPicko the vulnerabilities with the characteristics that we wanted to test.

Evaluating web vulnerability scanners. There exists a growing body of literature on the evaluation of web vulnerability scanners. For example, Suto compared three scanners against three different applications and used code coverage, among other metrics, as a measure of the effectiveness of each scanner [21]. In a recent follow-up study, Suto [22] assessed seven scanners and compared their detection capabilities and the time required to run them. Wiegenstein et al. ran five unnamed scanners against a custom benchmark [24]. Unfortunately, the authors do not discuss in detail the reasons for detections or spidering failures. In their survey of web security assessment tools, Curphey and Araujo reported that black-box scanners perform poorly [3]. Peine examined in depth the functionality and user interfaces of seven scanners (three commercial) that were run against WebGoat and one real-world application [16]. Kals et al. developed a new web vulnerability scanner and tested it on about 25,000 live web pages [7]. Since no ground truth is available for these sites, the authors cannot discuss false negative rate or failures of their tool. More recently, AnantaSec released an evaluation of three scanners against 13 real-world applications, three web applications provided by the scanners vendors, and a series of JavaScript tests [1]. While this experiment assesses a 
large number of real-world applications, only a limited number of scanners are tested and no explanation is given for the results. In addition, Vieira et al. tested four web scanners on 300 web services [23]. They also report high rates of false positives and false negatives.

In comparison, our work, to the best of our knowledge, performs the largest evaluation of web application scanners in terms of the number of tested tools (eleven, both commercial and open-source), and the class of vulnerabilities analyzed. In addition, we discuss the effectiveness of different configurations and levels of manual intervention, and examine in detail the reasons for a scanner's success or failure.

Furthermore, we provide a discussion of challenges (i.e., critical limitations) of current web vulnerability scanners. While some of these problem areas were discussed before $[6,8]$, we provide quantitative evidence that these issues are actually limiting the performance of today's tools. We believe that this discussion will provide useful insight into how to improve state-of-the-art of black-box web vulnerability scanners.

\section{Conclusions}

This paper presented the evaluation of eleven black-box web vulnerability scanners. The results of the evaluation clearly show that the ability to crawl a web application and reach "deep" into the application's resources is as important as the ability to detect the vulnerabilities themselves.

It is also clear that although techniques to detect certain kinds of vulnerabilities are well-established and seem to work reliably, there are whole classes of vulnerabilities that are not well-understood and cannot be detected by the state-of-the-art scanners. We found that eight out of sixteen vulnerabilities were not detected by any of the scanners.

We have also found areas that require further research so that web application vulnerability scanners can improve their detection of vulnerabilities. Deep crawling is vital to discover all vulnerabilities in an application. Improved reverse engineering is necessary to keep track of the state of the application, which can enable automated detection of complex vulnerabilities.

Finally, we found that there is no strong correlation between cost of the scanner and functionality provided as some of the free or very cost-effective scanners performed as well as scanners that cost thousands of dollars.

\section{Acknowledgments}

This work has been supported by the National Science Foundation, under grants CCR0524853, CCR-0716095, CCR-0831408, CNS-0845559 and CNS-0905537, and by the ONR under grant N000140911042.

\section{References}

1. AnantaSec: Web Vulnerability Scanners Evaluation (January 2009), http: //anantasec.blogspot.com/2009/01/web-vulnerability-scanners-comparison.html 
2. Balzarotti, D., Cova, M., Felmetsger, V., Vigna, G.: Multi-module Vulnerability Analysis of Web-based Applications. In: Proceedings of the ACM conference on Computer and Communications Security (CCS). pp. 25-35 (2007)

3. Curphey, M., Araujo, R.: Web Application Security Assessment Tools. IEEE Security and Privacy 4(4), 32-41 (2006)

4. CVE: Common Vulnerabilities and Exposures. http://www. cve.mitre.org

5. Foundstone: Hacme Bank v2.0. http://www. foundstone.com/us/resources/ proddesc/hacmebank . htm (May 2006)

6. Grossman, J.: Challenges of Automated Web Application Scanning. In: BlackHat Windows Security Conference (2004)

7. Kals, S., Kirda, E., Kruegel, C., Jovanovic, N.: SecuBat: A Web Vulnerability Scanner. In: Proceedings of the International World Wide Web Conference (2006)

8. McAllister, S., Kruegel, C., Kirda, E.: Leveraging User Interactions for In-Depth Testing of Web Applications. In: Proceedings of the Symposium on Recent Advances in Intrusion Detection (2008)

9. Open Security Foundation: OSF DataLossDB: Data Loss News, Statistics, and Research. http: //datalossdb.org/

10. Open Web Application Security Project (OWASP): OWASP SiteGenerator. http: / / www . owasp.org/index.php/OWASP \_SiteGenerator

11. Open Web Application Security Project (OWASP): OWASP WebGoat Project. http://www.owasp.org/index.php/Category:OWASP_WebGoat_Project

12. Open Web Application Security Project (OWASP): Web Input Vector Extractor Teaser. http://code.google.com/p/wivet/

13. Open Web Application Security Project (OWASP): OWASP Top Ten Project. http:// www. owasp.org/index.php/Top_10 (2010)

14. OpenID Foundation: OpenID. http://openid.net/

15. PCI Security Standards Council: PCI DDS Requirements and Security Assessment Procedures, v1.2 (October 2008)

16. Peine, H.: Security Test Tools for Web Applications. Tech. Rep. 048.06, Fraunhofer IESE (January 2006)

17. Provos, N., Mavrommatis, P., Rajab, M., Monrose, F.: All Your iFRAMEs Point to Us. In: Proceedings of the USENIX Security Symposium. pp. 1-16 (2008)

18. RSnake: Sql injection cheat sheet. http://ha.ckers.org/sqlinjection/

19. RSnake: XSS (Cross Site Scripting) Cheat Sheet. http://ha.ckers . org/xss . html

20. Small, S., Mason, J., Monrose, F., Provos, N., Stubblefield, A.: To Catch a Predator: A Natural Language Approach for Eliciting Malicious Payloads. In: Proceedings of the USENIX Security Symposium (2008)

21. Suto, L.: Analyzing the Effectiveness and Coverage of Web Application Security Scanners (October 2007), case Study

22. Suto, L.: Analyzing the Accuracy and Time Costs of Web Application Security Scanners (Feb 2010)

23. Vieira, M., Antunes, N., Madeira, H.: Using Web Security Scanners to Detect Vulnerabilities in Web Services. In: Proceedings of the Conference on Dependable Systems and Networks (2009)

24. Wiegenstein, A., Weidemann, F., Schumacher, M., Schinzel, S.: Web Application Vulnerability Scanners—a Benchmark. Tech. rep., Virtual Forge GmbH (October 2006) 